\title{
Adjuvant radiotherapy may have significant survival benefits for gastric cancer patients with 1-29 lymph nodes retrieved
}

\author{
Siyi Wu^, Yuxin $\mathrm{Chu}^{\wedge}$, Qinyong $\mathrm{Hu}^{\wedge}$, Qibin Song^ \\ Department of Oncology I, Cancer Center, Renmin Hospital of Wuhan University, Wuhan, China \\ Contributions: (I) Conception and design: Q Hu; (II) Administrative support: Q Song; (III) Provision of study materials or patients: Y Chu; (IV) \\ Collection and assembly of data: S Wu; (V) Data analysis and interpretation: Y Chu; (VI) Manuscript writing: All authors; (VII) Final approval of \\ manuscript: All authors. \\ Correspondence to: Qibin Song. Department of Oncology I, Cancer Center, Renmin Hospital of Wuhan University, Jiefang Road No. 238, Wuhan \\ 430060, China. Email: qibinsong@whu.edu.cn.
}

\begin{abstract}
Background: The role of adjuvant radiotherapy (RT) in gastric cancer (GC) patients has not been wellestablished. This study initiated a retrospective case-control study to explore the survival impact of adjuvant RT on these patients.

Methods: All patients were collected from the Surveillance, Epidemiology, and End Results (SEER) database. The cohort was assigned into patients without adjuvant RT versus those with adjuvant RT. Descriptive chi-square test was adopted to compare categorical variates between the 2 groups. Kaplan-Meier (KM) method was adopted to estimate the patients' cancer-specific survival (CSS) and overall survival (OS). Cox proportional hazard models were utilized to characterize the prognostic factors of their CSS.

Results: Totally 7,194 patients were recruited in this study, 3,326 (46.2\%) patients didn't have RT and $3,868(53.8 \%)$ patients had adjuvant RT. Survival analysis of the entire population showed that adjuvant RT had remarkable survival benefits for the GC patients. The median CSS was 47.0 (42.0-52.0) months in RT group versus 32.0 (29.7-34.3) months in no RT group $(\mathrm{P}<0.001)$. Age $>60$, histologic type 8490, tumor size $>50 \mathrm{~mm}$, higher stage TNM, and surgery type 40/50 were independent risk factors for poor prognosis. Comparatively, adjuvant RT and LN examined $>0$ were independent factors for improving prognosis. Subgroup analysis demonstrated that adjuvant RT had significant survival benefits for patients with 1-14 and 15-29 lymph nodes (LNs) retrieved.
\end{abstract}

Conclusions: Adjuvant radiotherapy may have significant survival benefits for GC patients with 1-29 LNs retrieved. Our study upholds adjuvant RT for this subset of patients.

Keywords: Radiotherapy (RT); gastric cancer (GC); Surveillance, Epidemiology, and End Results (SEER); survival

Submitted Apr 02, 2020. Accepted for publication Sep 24, 2020.

doi: $10.21037 /$ tcr-20-1750

View this article at: http://dx.doi.org/10.21037/tcr-20-1750

\section{Introduction}

As an important malignancy worldwide, gastric cancer (GC) has become the fifth most frequent cancer, over 1,000,000 new cases were diagnosed in 2018 , and approximately
783,000 deaths (1:12 deaths globally) were estimated, making it the third leading cause of cancer-related death (1). Unfortunately, $35 \%$ of GC patients have distant metastasis at preliminary diagnosis, with unfavorable prognosis (2).

\footnotetext{
^ Siyi Wu: ORCID: 0000-0001-7195-4141; Yuxin Chu: ORCID: 0000-0001-5526-997X; Qinyong Hu: ORCID: 0000-0002-0764-3792; Qibin Song: ORCID: 0000-0003-2387-6438.
} 
The overwhelming majority (about $95 \%$ ) of GC are adenocarcinomas (3), most patients are diagnosed at late stages (4). Consequently, the prognosis of GC patients is usually exacerbated by prevalent metastasis or recurrence, making it a big challenge for clinicians in terms of cancer treatment.

The most effective treatment for GC with curative intent is surgical resection, combining with neoadjuvant or adjuvant chemotherapy/radiotherapy, to curb the disease and prolong survival. The SWOG-9008/INT0116 trial has indicated the survival superiority of postoperative chemoradiotherapy (CRT) to surgery alone, but $90 \%$ of selected patients received D0 or D1 lymphadenectomy (5). It was criticized that adjuvant CRT only compensated for patients with non-radical surgery. Additionally, a latest study also evaluated the effect of adjuvant radiotherapy (RT) in the patients with intestinal-type gastric adenocarcinoma. It concluded that adjuvant RT had significant survival benefits for such patients (6). However, the ARTIST trial revealed that, in GC patients after D2 lymph node (LN) dissection, neither CRT nor chemotherapy showed survival benefits, except for the LN+ subgroup of patients in 3-year survival (7). So far, it's still inconclusive regarding the value of CRT compared to chemotherapy or no treatment (8). The evidence of distinct benefit from adjuvant $\mathrm{RT}$ is deficient in the postoperative setting for GC (9). The extent of lymphadenectomy may also affect the survival benefits from adjuvant RT, but the optimal adjuvant strategy for GC patients still remains elusive.

With a big population of GC patients from the Surveillance, Epidemiology and End Results (SEER) database, we retrospectively analyzed the clinical characteristics of these patients after palliative gastrectomy with chemotherapy. We aimed to elucidate whether adjuvant RT could exert additional survival advantage for such patients. We also identified the prognostic factors associated with the patients' cancer specific survival (CSS), in order to characterize which subgroup of patients would acquire optimal survival benefits from adjuvant RT (10). We present the following article in accordance with the STROBE reporting checklist (available at http://dx.doi. org/10.21037/tcr-20-1750).

\section{Methods}

The study conformed to the provisions of the Declaration of Helsinki (as revised in 2013).

\section{Patient selection}

All the patients were extracted from the SEER 18 registries Custom Database. We selected the patients via SEER*Stat software. We comply with the Data-Use Agreement, so we are entitled to access the SEER data. The SEER database is openly accessible, so we don't need the informed consent from patients (11). All the cases were recruited and analyzed anonymously. This study conformed to the ethical criteria. We initiated a retrospective case-control study. The inclusion criteria were listed below: (I) the diagnosis was between 2004 and 2015; (II) only one primary site at the stomach; (III) GC was the sole or first cancer; (IV) gastrectomy was performed; (V) chemotherapy recode was "yes"; (VI) radiation sequence was "No radiation and/or cancer-directed surgery" or "Radiation after surgery". The patients were pathologically confirmed GC. The exclusion criteria for enrolled patients were: (I) missing information on age, race, grade, stage; (II) benign or borderline tumors; (III) unknown survival data.

\section{Data collection}

We chose these table variables: gender, age, race, histologic type, tumor size, grade, TNM stage, tumor depth, LN metastasis, distant metastasis, LN examined, surgery type, radiation status, survival months, cause-specific death, and vital status. In our analysis, the primary endpoint is CSS, denoting the time from diagnosis to mortality from GC. The secondary endpoint is overall survival (OS), defining the span from cancer diagnosis to death from any reason.

\section{Statistical analysis}

All the patients were assigned into those without versus those with adjuvant RT. The patients' clinical characteristics were described with chi-square test. The CSS and OS differences between the 2 groups were displayed by KaplanMeier (KM) plots with log-rank test. The predictors of patients' CSS were identified by univariate and multivariate Cox proportional hazards regression model. Stratified analysis was conducted according to the independent predictors. The SPSS 25.0 software was used to conduct statistical analyses. The $\mathrm{P}<0.05$ (two-sided probability) was 
deemed statistically significant.

\section{Results}

\section{Patient features}

Totally 7,194 patients were finally included in analysis, of whom 3,326 (46.2\%) cases didn't have RT and 3,868 (53.8\%) cases had adjuvant RT. In descriptive statistics, except gender, histologic type, and grade $(\mathrm{P}>0.05)$, other variables showed remarkable differences between no RT and RT groups $(\mathrm{P}<0.05)$. In comparison with the no RT group, the RT group had more patients with age $\leq 60(45.6 \%$ vs. $43.4 \%)$, black race (15.9\% vs. $13.8 \%)$. As for tumor features, more patients in the RT group were tumor size $\leq 50 \mathrm{~mm}$, stage II-III, T2-3, N1-2, M1, and LN examined $=1-29$ (all $\mathrm{P}<0.05)$. Additionally, the RT group was also more likely to receive partial gastrectomy or gastrectomy with removal of a portion of esophagus. The clinical variables of patients in the 2 comparison groups are listed in Table 1.

\section{Survival analysis of entire cohort}

With the entire population, the survival between no RT and RT cohort was compared by Kaplan-Meier method with log-rank test. The plots showed the RT group behaved significantly better prognosis than the control group in both CSS and OS (Figure 1A,B, $\mathrm{P}<0.05$ ). The median CSS

Table 1 The clinical variables of the patients after gastrectomy $(n=7,194)$

\begin{tabular}{|c|c|c|c|c|}
\hline Variable & No RT, n (\%) & RT, n (\%) & Total, n (\%) & $P$ value \\
\hline$N(\%)$ & $\mathrm{n}=3,326(46.2)$ & $\mathrm{n}=3,868(53.8)$ & $\mathrm{n}=7,194(100.0)$ & \\
\hline Gender & & & & 0.920 \\
\hline Male & $2,090(62.8)$ & 2,435 (63.0) & $4,525(62.9)$ & \\
\hline Female & $1,236(37.2)$ & $1,433(37.0)$ & 2,669 (37.1) & \\
\hline Age (years) & & & & 0.032 \\
\hline$\leq 60$ & $1,445(43.4)$ & $1,763(45.6)$ & $3,208(44.6)$ & \\
\hline $60-80$ & $1,665(50.1)$ & $1,902(49.2)$ & 3,567 (49.6) & \\
\hline$>80$ & $216(6.5)$ & $203(5.2)$ & $419(5.8)$ & \\
\hline Race & & & & $<0.001$ \\
\hline White & $2,183(65.6)$ & $2,333(60.3)$ & $4,516(62.8)$ & \\
\hline Black & $458(13.8)$ & $616(15.9)$ & $1,074(14.9)$ & \\
\hline Others & $685(20.6)$ & $919(23.8)$ & $1,604(22.3)$ & \\
\hline Histologic type & & & & 0.555 \\
\hline 8140 & $1,416(42.6)$ & $1,641(42.4)$ & 3,057 (42.5) & \\
\hline 8490 & $830(25.0)$ & 964 (24.9) & $1,794(24.9)$ & \\
\hline 8144 & $474(14.3)$ & $534(13.8)$ & $1,008(14.0)$ & \\
\hline 8145 & $230(6.9)$ & $307(7.9)$ & $537(7.5)$ & \\
\hline Other & $376(11.3)$ & $422(10.9)$ & 798 (11.1) & \\
\hline Tumor size (mm) & & & & $<0.001$ \\
\hline$\leq 50$ & $1,549(46.6)$ & 2,025 (52.4) & $3,574(49.7)$ & \\
\hline$>50$ & $1,777(53.4)$ & $1,843(47.6)$ & $3,620(50.3)$ & \\
\hline
\end{tabular}

Table 1 (continued) 
Table 1 (continued)

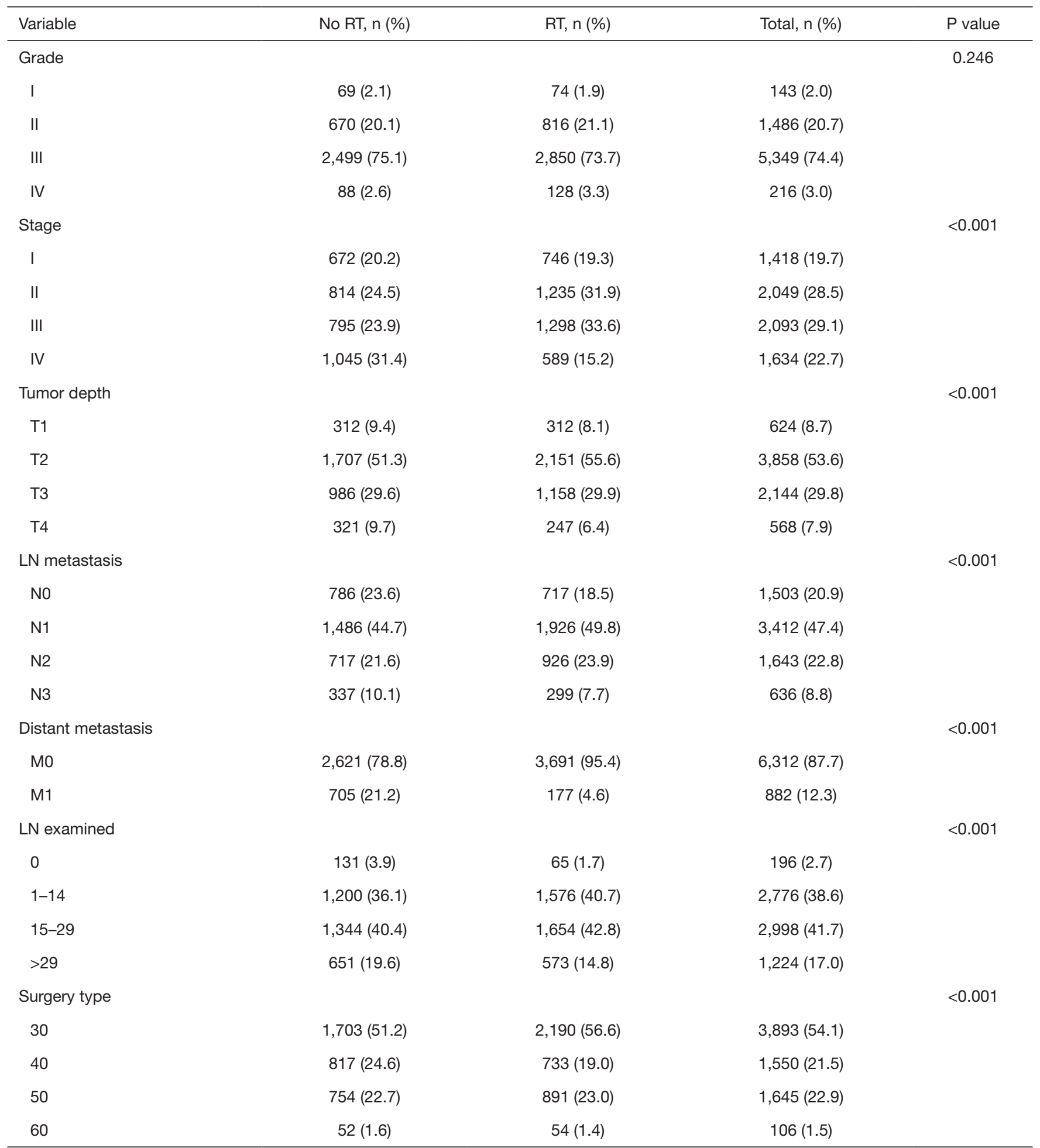

8140-Adenocarcinoma; 8490-Signet ring cell carcinoma; 8144-Adenocarcinoma, intestinal type; 8145-Adenocarcinoma, diffuse type. 30-Gastrectomy (partial, subtotal, hemi-); 40-Near-total or total gastrectomy; 50-Gastrectomy with removal of a portion of esophagus; $60-$ Gastrectomy with a resection in continuity with the resection of other organs. LN, lymph node; RT, radiotherapy. 

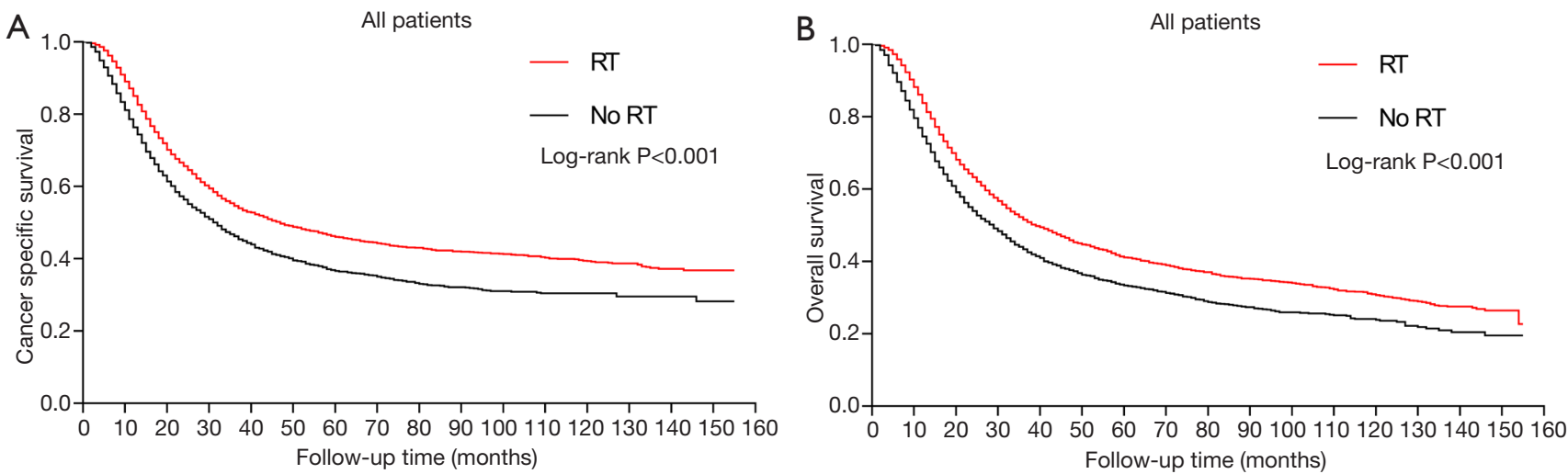

Figure 1 Kaplan-Meier survival curves for the entire cohort. (A) CSS $(\mathrm{P}<0.001)$. (B) $\mathrm{OS}(\mathrm{P}<0.001)$. RT, radiotherapy; CSS, cancer specific survival; OS, overall survival.

Table 2 Comparison of the patients' median survival $(\mathrm{n}=7,194)$

\begin{tabular}{lccc}
\hline Treatment & Patients, No. & $\begin{array}{c}\text { Median CSS, } \\
95 \% \mathrm{Cl} \text {, months }\end{array}$ & $\begin{array}{c}\text { Median OS, } \\
95 \% \mathrm{Cl} \text {, months }\end{array}$ \\
\hline No radiation & 3,326 & $32.0(29.7-34.3)$ & $29.0(27.1-30.9)$ \\
Radiation & 3,868 & $47.0(42.0-52.0)$ & $39.0(36.2-41.8)$ \\
$\mathrm{P}$ value & & $<0.001$ & $<0.001$ \\
\hline
\end{tabular}

No., number; CSS, cancer specific survival; OS, overall survival; $\mathrm{Cl}$, confidence interval.

in RT cohort was 47.0 (42.0-52.0) months, while that of no RT group was 32.0 (29.7-34.3) months (Table 2, $\mathrm{P}<0.05$ ). Similarly, the RT group also had significantly superior median OS than the no RT group, median OS for RT cohort was 39.0 vs. 29.0 months for no RT cohort (Table 2, $\mathrm{P}<0.001)$. These results demonstrated that adjuvant RT had significant survival benefits for the GC patients as a whole.

\section{Identify prognostic factors}

We utilized the Cox regression models to determine the predictors of patients' CSS. As for univariate Cox analysis, except gender, other variables were all significantly associated with the CSS $(\mathrm{P}<0.05)$. Those significant variates were further brought into multivariate Cox model. After adjusting the confounding factors, age $>60$, histologic type 8490 , tumor size $>50 \mathrm{~mm}$, higher stage TNM, and surgery type $40 / 50$ were identified as independent predictors for unfavorable prognosis $(\mathrm{HR}>1, \mathrm{P}<0.05)$. By comparison, receiving adjuvant $\mathrm{RT}$ and $\mathrm{LN}$ examined $>0$ were independent predictors for improving prognosis $(\mathrm{HR}<1, \mathrm{P}<0.05)$. Concretely, adjuvant RT still decreased the mortality risk from cancer $(\mathrm{HR}=0.846 ; 95 \% \mathrm{CI}$, $0.791-0.905, \mathrm{P}<0.001)$. Hence adjuvant $\mathrm{RT}$ is identified as an independent protective variable in the GC patients after gastrectomy. The specific outcome can be seen in Table 3 .

\section{Stratified analysis}

In Cox regression analyses, $\mathrm{LN}$ examined was also identified as an independent protective factor, so we initiated a stratified analysis to emphasize the effect of adjuvant RT on the CSS of patients. The stratified plots displayed that the survival difference was not significant between no RT and RT group in patients with either $\mathrm{LN}$ examined $=0$ or $>29$ (Figure $2 A, B, \mathrm{P}>0.05$ ). However, adjuvant RT exerted remarkable CSS benefits for the patients with $\mathrm{LN}$ examined $=1-14$ and 15-29 (Figure $2 C, D, \mathrm{P}<0.001$ ). Thus, adjuvant radiation may have remarkable survival advantages for the GC patients with 1-29 LNs retrieved.

\section{Discussion}

This study is intended to validate the efficacy of adjuvant RT, with respect to CSS and OS, for GC patients after palliative gastrectomy with chemotherapy. The overall results indicated that adjuvant RT exerted superior CSS and OS for these patients. The multivariate Cox regression analyses identified adjuvant $\mathrm{RT}$ as an independent protective factor for CSS. Additionally, subgroup survival analysis demonstrated that only patients with 1-14 and 1529 LNs examined acquired significant survival benefits from adjuvant RT.

Several previous studies have reported the effect of 
Table 3 Cox regression analysis for cancer specific survival $(n=7,194)$

\begin{tabular}{|c|c|c|c|c|}
\hline Variable & \multicolumn{2}{|c|}{ Univariate cox } & \multicolumn{2}{|c|}{ Multivariate cox } \\
\hline \multicolumn{5}{|l|}{$\mathrm{RT}$} \\
\hline No & Reference & & Reference & \\
\hline Yes & $0.747(0.701-0.796)$ & $<0.001$ & $0.846(0.791-0.905)$ & $<0.001$ \\
\hline Male & Reference & & $\mathrm{NI}$ & \\
\hline Female & 1.057 (0.991-1.128) & 0.092 & & \\
\hline \multicolumn{5}{|c|}{ Age (years) } \\
\hline$\leq 60$ & Reference & & Reference & \\
\hline \multicolumn{5}{|l|}{ Race } \\
\hline White & Reference & & Reference & \\
\hline Black & $0.967(0.883-1.058)$ & 0.462 & $1.003(0.915-1.100)$ & 0.942 \\
\hline Others & $0.809(0.746-0.876)$ & $<0.001$ & $0.837(0.771-0.908)$ & $<0.001$ \\
\hline \multicolumn{5}{|c|}{ Histologic type } \\
\hline 8140 & Reference & & Reference & \\
\hline 8490 & $1.317(1.22-1.422)$ & $<0.001$ & $1.181(1.089-1.281)$ & $<0.001$ \\
\hline 8144 & $0.838(0.756-0.930)$ & 0.001 & $0.975(0.877-1.084)$ & 0.646 \\
\hline \multicolumn{5}{|l|}{ Grade } \\
\hline I & Reference & & Reference & \\
\hline II & $1.158(0.871-1.54)$ & 0.313 & $1.056(0.793-1.407)$ & 0.707 \\
\hline III & $1.848(1.401-2.437)$ & $<0.001$ & $1.378(1.042-1.823)$ & 0.025 \\
\hline IV & $2.266(1.643-3.125)$ & $<0.001$ & $1.584(1.145-2.191)$ & 0.005 \\
\hline \multicolumn{5}{|l|}{ Stage } \\
\hline I & Reference & & Reference & \\
\hline II & $2.008(1.776-2.271)$ & $<0.001$ & $1.464(1.234-1.736)$ & $<0.001$ \\
\hline III & $3.600(3.200-4.051)$ & $<0.001$ & $1.936(1.591-2.355)$ & $<0.001$ \\
\hline IV & $6.373(5.658-7.179)$ & $<0.001$ & $2.233(1.765-2.824)$ & $<0.001$ \\
\hline
\end{tabular}

Table 3 (continued) 
Table 3 (continued)

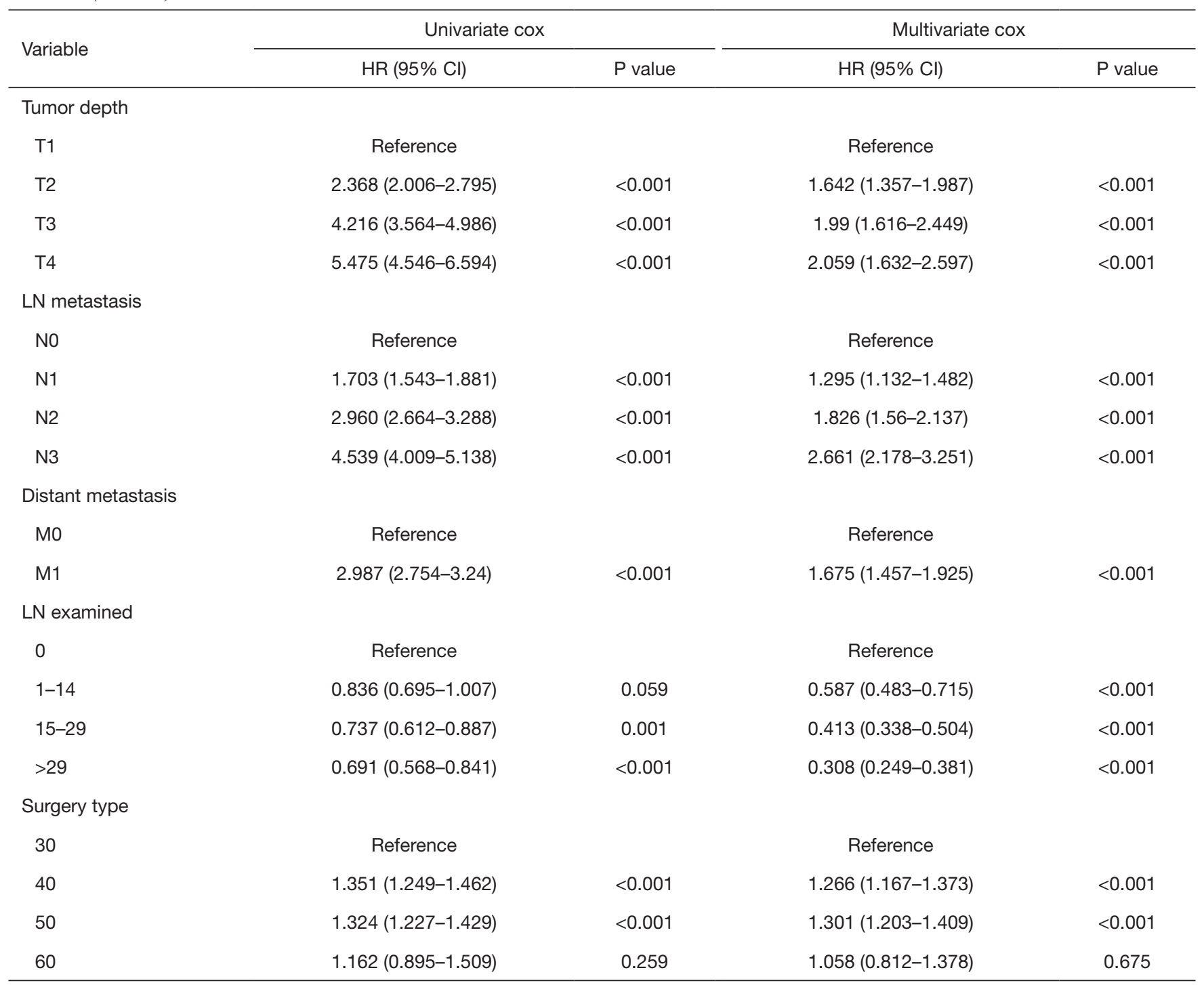

8140-Adenocarcinoma; 8490-Signet ring cell carcinoma; 8144-Adenocarcinoma, intestinal type; 8145-Adenocarcinoma, diffuse type. 30-Gastrectomy (partial, subtotal, hemi-); 40-Near-total or total gastrectomy; 50-Gastrectomy with removal of a portion of esophagus; $60-$ Gastrectomy with a resection in continuity with the resection of other organs. HR, hazard ratio; $\mathrm{Cl}$, confidence interval; NI, not included; LN, lymph node; RT, radiotherapy.

adjuvant RT on patients with resectable GC. A retrospective study has evaluated the influence of postoperative CRT on the patients with D2-dissected GC. Its overall results indicated that, the median OS of the CRT group was 51.0 months, while that in CT group was 48.6 months ( $\mathrm{P}>0.05)$. In parallel, the median DFS was 40.7 vs. 31.2 months $(\mathrm{P}>0.05)$. This study collected a total of 337 eligible patients, the power of analysis may be limited by small sample size (12). Another study has compared the OS between perioperative chemotherapy and adjuvant CRT in gastric adenocarcinoma patients from the NCDB database. The results showed that adjuvant CRT had significant OS advantages over chemotherapy alone, the median OS was 49 vs. 39 months $(\mathrm{P}<0.05)(13)$. They concluded that addition of adjuvant radiotherapy was advantageous for resected GC. However, the impact of postoperative radiation on the CSS 

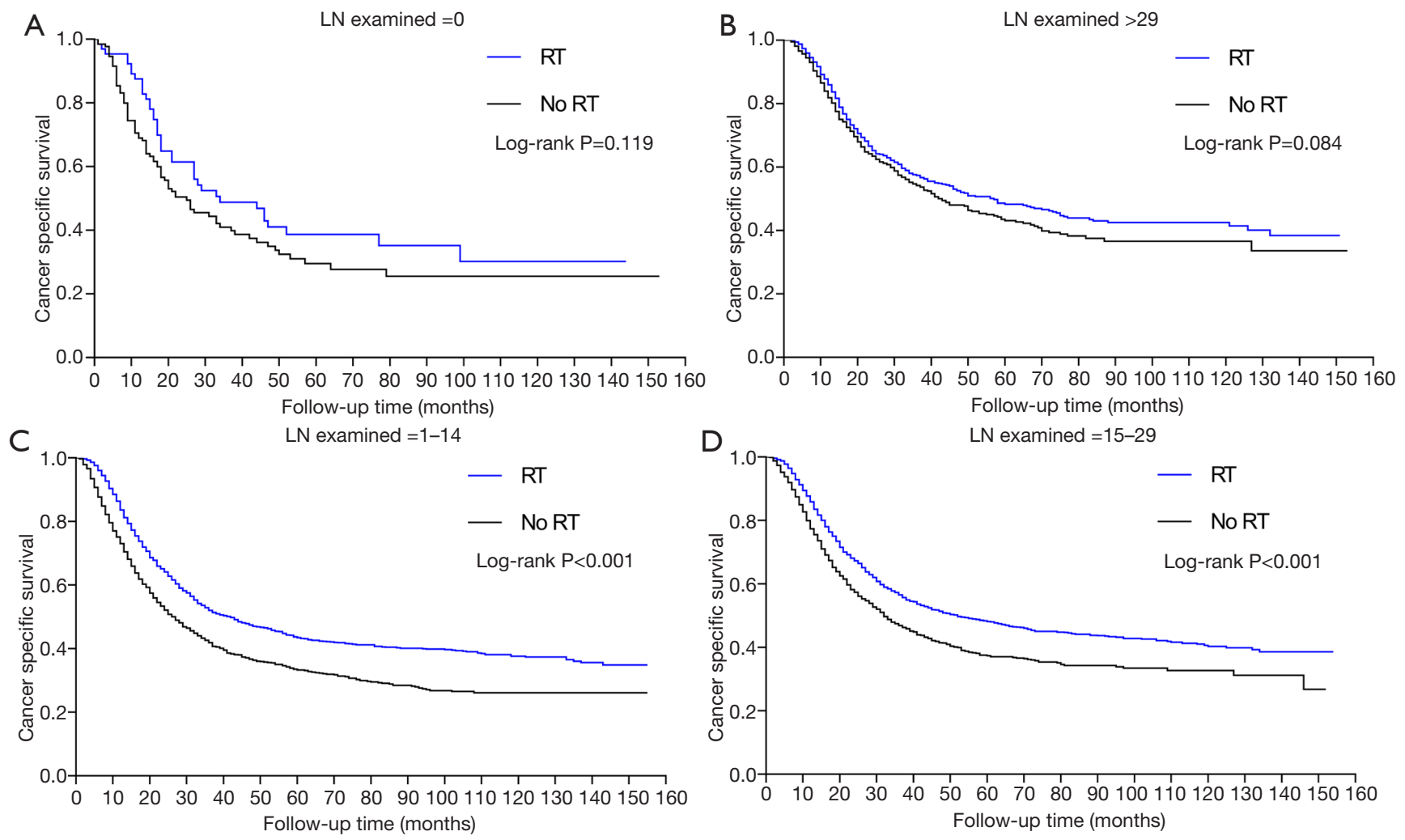

Figure 2 Kaplan-Meier survival curves for CSS stratified by LN examined. (A) LN examined $=0$ ( $>>0.05)$. (B) LN examined $>29$ (P>0.05). (C) LN examined $=1-14(\mathrm{P}<0.001)$. (D) LN examined =15-29 ( $<<0.001)$. LN, lymph node; RT, radiotherapy; CSS, cancer specific survival.

of recruited patients was not indicated. Furthermore, the CRITICS study demonstrated that, in patients treated with adequate surgery and preoperative chemotherapy, adjuvant CRT showed no OS advantage in comparison with adjuvant chemotherapy alone. This study had a population bias, because only half of the patients accomplished the whole study (14). Comparatively, our study recruited a much larger sample size from the SEER database. In our results, the median OS in RT cohort was 39.0 vs. 29.0 months in no RT cohort $(\mathrm{P}<0.001)$. Similarly, the median CSS was 47.0 (42.0-52.0) vs. $32.0(29.7-34.3)$ months $(\mathrm{P}<0.001)$. Therefore, significant survival benefits from adjuvant RT were found for the entire GC patients.

Regarding the prognostic factors of GC, a recent article has identified T-stage, $\mathrm{N}$-status, nodal ratio, and other histological factors as critical variables when considering postoperative combined treatment for such patients (15). Consistently, our multivariate Cox regression analysis has also identified age $>60$, histologic type 8490 , tumor size $>50 \mathrm{~mm}$, higher stage TNM, and surgery type 40/50 as independent risk factors, while adjuvant RT and LN examined $>0$ as independent protective factors. These prognostic factors will become helpful for selecting which subgroup of patients may be more appropriate for adjuvant RT.

Based on a large cohort from the international database, a recent study declared that lymphadenectomy with optimum of 29 LNs retrieved might improve the survival of advanced GC (16). However, the effect of adjuvant RT on these patients still remained unclear. According to our subgroup survival analysis, adjuvant RT only benefited the CSS of patients with 1-29 LNs examined. This subset of patients may get more survival benefits from adjuvant RT.

Several limitations should be concerned. First, this retrospective study design may have inherent selection bias, because the variables in our analysis are selected from previous data (17). Second, the patient comorbidities, chemotherapy regime, surgical margin status, and possible complications after surgery are not available in the SEER database (18). Third, several important radiation parameters are not listed in the SEER database, such as the radiation doses, fields, and radiation ranges. When considering the 
submission of adjuvant RT for GC in clinical settings, adequate cautions should still be concerned. Given those limitations stated above, we will design some prospective studies to overcome the bias in the future. Moreover, we will also collect patient information from our hospital, such as chemotherapy regime, complications, and radiation parameters, in order to validate our findings.

\section{Conclusions}

This retrospective study has suggested that adjuvant RT may have significant survival benefits for GC patients with 1-29 LNs retrieved. Our report will provide more information to support adjuvant RT for these patients.

\section{Acknowledgments}

The authors acknowledge the Surveillance, Epidemiology, and End Results database for providing high quality clinical data for our study.

Funding: This study was supported by the National Natural Science Foundation of China [grant no. 81670123, 81670144].

\section{Footnote}

Reporting Checklist: The authors have completed the STROBE guideline checklist. Available at http://dx.doi. org/10.21037/tcr-20-1750

Conflicts of Interest: All authors have completed the ICMJE uniform disclosure form (available at http://dx.doi. org/10.21037/tcr-20-1750). The authors have no conflicts of interest to declare.

Ethical Statement: The authors are responsible for all aspects of the work, ensuring that questions related to the accuracy or integrity of any part of the work are appropriately investigated and resolved. The study conformed to the provisions of the Declaration of Helsinki (as revised in 2013).

Open Access Statement: This is an Open Access article distributed in accordance with the Creative Commons Attribution-NonCommercial-NoDerivs 4.0 International License (CC BY-NC-ND 4.0), which permits the noncommercial replication and distribution of the article with the strict proviso that no changes or edits are made and the original work is properly cited (including links to both the formal publication through the relevant DOI and the license). See: https://creativecommons.org/licenses/by-nc-nd/4.0/.

\section{References}

1. Bray F, Ferlay J, Soerjomataram I, et al. Global cancer statistics 2018: GLOBOCAN estimates of incidence and mortality worldwide for 36 cancers in 185 countries. CA Cancer J Clin 2018;68:394-424.

2. Picado O, Dygert L, Macedo FI, et al. The Role of Surgical Resection for Stage IV Gastric Cancer With Synchronous Hepatic Metastasis. J Surg Res 2018;232:422-9.

3. Perrot-Applanat M, Vacher S, Pimpie C, et al. Differential gene expression in growth factors, epithelial mesenchymal transition and chemotaxis in the diffuse type compared with the intestinal type of gastric cancer. Oncol Lett 2019;18:674-86.

4. Kim KY, Yi BR, Lee HR, et al. Stem cells with fused gene expression of cytosine deaminase and interferon- $\beta$ migrate to human gastric cancer cells and result in synergistic growth inhibition for potential therapeutic use. Int J Oncol 2012;40:1097-104.

5. Macdonald JS, Smalley SR, Benedetti J, et al. Chemoradiotherapy after surgery compared with surgery alone for adenocarcinoma of the stomach or gastroesophageal junction. N Engl J Med 2001;345:725-30.

6. Chu Y, Xu B, Song Q, et al. Radiotherapy benefited the survival of patients with intestinal-type gastric adenocarcinoma: a SEER population-based study. Clin Transl Oncol 2020. [Epub ahead of print].

7. Lee J, Lim DH, Kim S, et al. Phase III trial comparing capecitabine plus cisplatin versus capecitabine plus cisplatin with concurrent capecitabine radiotherapy in completely resected gastric cancer with $\mathrm{D} 2$ lymph node dissection: the ARTIST trial. J Clin Oncol 2012;30:268-73.

8. Ho VKY, Jansen EPM, Wijnhoven BPL, et al. Adjuvant Chemoradiotherapy for Non-Pretreated Gastric Cancer. Ann Surg Oncol 2017;24:3647-57.

9. Xu J, Zhu J, Wei Q. Adjuvant Radiochemotherapy versus Chemotherapy Alone for Gastric Cancer: Implications for Target Definition. J Cancer 2019;10:458-66.

10. Ma GF, Zhang HG, Liu J, et al. Benefit of adjuvant chemoradiotherapy in patients with pathological stage III gastric cancer. Cancer Manag Res 2019;11:6029-41.

11. Zhang C, Liu L, Tao F, et al. Bone Metastases Pattern in Newly Diagnosed Metastatic Bladder Cancer: A 
Population-Based Study. J Cancer 2018;9:4706-11.

12. Peng J, Wei Y, Zhou F, et al. D2-resected stage IIIc gastric cancer patients benefit from adjuvant chemoradiotherapy. Cancer Med 2016;5:2773-80.

13. Stumpf PK, Amini A, Jones BL, et al. Adjuvant radiotherapy improves overall survival in patients with resected gastric adenocarcinoma: A National Cancer Data Base analysis. Cancer 2017;123:3402-9.

14. Cats A, Jansen E, Grieken N, et al. Chemotherapy versus chemoradiotherapy after surgery and preoperative chemotherapy for resectable gastric cancer (CRITICS): an international, open-label, randomised phase 3 trial. Lancet Oncol 2018;19:616-28.

15. Agolli L, Maurizi Enrici R, Osti MF. Adjuvant radiochemotherapy for gastric cancer: Should we

Cite this article as: $\mathrm{Wu} \mathrm{S,} \mathrm{Chu} \mathrm{Y,} \mathrm{Hu} \mathrm{Q,} \mathrm{Song} \mathrm{Q.} \mathrm{Adjuvant}$ radiotherapy may have significant survival benefits for gastric cancer patients with 1-29 lymph nodes retrieved. Transl Cancer Res 2020;9(11):6929-6938. doi: 10.21037/tcr-20-1750 use prognostic factors to select patients? World J

Gastroenterol 2016;22:1131-8.

16. Woo Y, Goldner B, Ituarte P, et al. Lymphadenectomy with Optimum of 29 Lymph Nodes Retrieved Associated with Improved Survival in Advanced Gastric Cancer: A 25,000-Patient International Database Study. J Am Coll Surg 2017;224:546-55.

17. Fisher BW, Fluck M, Young K, et al. Urgent Surgery for Gastric Adenocarcinoma: A Study of the National Cancer Database. J Surg Res 2020;245:619-28.

18. Shridhar R, Almhanna K, Hoffe SE, et al. Increased survival associated with surgery and radiation therapy in metastatic gastric cancer: a Surveillance, Epidemiology, and End Results database analysis. Cancer 2013;119:1636-42. 